Revista Destaques Acadêmicos, Lajeado, v. 10, n. 3, 2018. ISSN 2176-3070

DOI: http://dx.doi.org/10.22410/issn.2176-3070.v10i3a2018.1960

http://www.univates.br/revistas

\title{
SÍNDROME DE BURNOUT EM ENFERMEIROS DE UNIDADE DE TERAPIA INTENSIVA: PRODUÇÃO CIENTÍFICA DE ENFERMAGEM
}

\author{
Jacquelane Silva Santos ${ }^{1}$, Lucas Barreto Pires Santos², \\ Jocimara Rodrigues de Lima ${ }^{3}$
}

\begin{abstract}
Resumo: O estudo objetivou revisar a produção científica nacional contextualizando a Síndrome de Burnout em enfermeiros de UTI. Método: trata-se de uma revisão integrativa de literatura, mediante pesquisa bibliográfica eletrônica, realizada entre maio e julho de 2017, na Biblioteca Virtual de Saúde (BVS) a partir de periódicos indexados na base de dados LILACS, IBECS, MEDLINE, BDENF e Index Psicologia. Resultados: foram localizados 73 artigos relacionados à temática, dos quais, cinco preencheram aos critérios de inclusão estabelecidos. Os estudos analisados evidenciaram que os profissionais de enfermagem que atuam em UTI apresentaram risco para desenvolver a Síndrome de Burnout devido a condições estressoras na qual estão impostas. Conclusão: o profissional de enfermagem precisa reconhecer seus limites de trabalho na Unidade de Terapia Intensiva (UTI).
\end{abstract}

Palavras-chave: Enfermeiros. Burnout. Unidade de terapia intensiva.

\section{INTRODUÇÃO}

A unidade hospitalar é caracterizada como ambiente de risco ocupacional aos indivíduos que trabalham nesta instituição. A organização do trabalho exige do profissional cada vez mais responsabilidade e conhecimentos tecnológicos, adaptáveis e reajustados a necessidade do serviço. A implementação de tecnologia na assistência é consequência da globalização gerando benefícios na sociedade e modificações que acarretam no comportamento biopsicossocial do ser humano (SILVA et al., 2014).

1 Enfermeira. Mestranda em enfermagem. Universidade de Pernambuco.

2 Enfermeiro. Universidade Federal da paraíba - UFPB.

3 Enfermeira. Universidade Federal de Alagoas - UFAL. 
Os enfermeiros são os profissionais da área de saúde que mantêm uma maior aproximação com pacientes e familiares, estando envolvidos com os aspectos emocionais, estresses e outros sentimentos apresentados pelos familiares dos pacientes (PEREIRA et al., 2014). Além disso, a demanda advinda do exercício profissional, como necessidade de atualizações, capacitações, diferentes condições de trabalho, novas configurações organizacionais, provocam consequentemente o desgaste físico, psíquico, emocional, caracterizando-se como fatores que propiciam o surgimento da Sindrome de Burnout (SCHMIDT et al., 2013).

No contexto hospitalar destaca-se a Unidade de Terapia Intensiva (UTI), que tem como propósito prestar assistência a pacientes gravemente acometidos, com cuidados intensivos, que requerem suportes tecnológicos, habilidades técnicas específicas, empatia com familiar do doente, envolvimento com o quadro clínico e prontidão de uma equipe especializada (MARTINS et al., 2014).

Os profissionais que atuam na UTI necessitam de agilidade para intervir em todas as situações, com precaução nos procedimentos para uma assistência em saúde segura e de qualidade. Reconhecer seu momento de bem-estar físico e emocional auxilia nas necessidades de cuidado ao paciente, ajuda na relação de confiança e contribui para minimizar eventos estressores que prejudicam sua rotina de trabalho (DECEZARO et al., 2014).

Enfermeiros que trabalham em UTI, por estarem mais expostos às situações de morte e luto, pacientes em estado grave ou paliativo, apresentam maior risco para desenvolver a Síndrome de Burnout (FERNANDES, NITSCHE, GODOY, 2015).

As exigências impostas pela complexidade do cuidado nessas unidades podem superar o limite do profissional, induzindo-o ao estresse prolongado que, em certas condições, emergem sofrimentos relacionados à sua história individual. Os desgastes que as pessoas podem obter no ambiente e relações de trabalho implicam no surgimento de doenças, onde os comportamentos adaptativos psicológicos como mecanismo de enfrentamento ficam debilitados (SILVA et al., 2015).

Quando a prevalência dos sintomas psíquicos interfere no desenvolvimento profissional, comprometendo as adaptações individuais, pode evoluir para a Síndrome de Burnout. Tal síndrome é caracterizada como transtorno adaptativo crônico, que está associado às exigências laborais e suas demandas, estresse e esgotamento físico psíquico e emocional crônico do indivíduo (ALKIMIM et al., 2014).

Entender a complexidade da Síndrome requer sensibilidade do profissional de saúde, visto que pode ser confundida com cansaço e estresse, desse modo, é necessário considerar principalmente os aspectos psíquicos e emocionais, observando o tempo de mudança de comportamento e demais 
alterações que contribuem para o diagnóstico. O rastreio na busca de situações conflitantes e identificação da característica individual dos profissionais contribui na prevenção dessa síndrome (FERREIRA, ARAGÃO, OLIVEIRA, 2017).

Os profissionais de saúde necessitam compreender o trabalho como espaço colaborativo do cuidado em saúde que tenha significado de saúde e não de doença. A identificação de agentes perturbadores do equilíbrio do profissional envolvido na assistência ao paciente contribui para implementação de intervenções eficazes. O reconhecimento tardio da Síndrome de Burnout e quando tratada de forma inadequada, pode levar à morte (CARVALHAIS et al., 2015).

A saúde do trabalhador, diante da complexidade e fragmentação das organizações do trabalho, deve ser estudada sob uma ótica interdisciplinar. Para isso, a psicodinâmica do Trabalho torna-se uma abordagem apropriada na tentativa de desvendar a dinâmica das situações de trabalho e os possíveis agravos à saúde do trabalhador. Entende-se que a atividade produtiva pode repercutir positiva ou negativamente no trabalhador (CAMPOS, DAVID, SOUZA, 2014).

Estudos mostram que os profissionais de enfermagem encontram-se suscetíveis as altas taxas de Burnout. Tal fato faz com que este estudo implique numa significativa relevância por fornecer dados que permitirão novos debates sobre o tema no meio acadêmico possibilitando a inserção de medidas em âmbito institucional que busquem reduzir os fatores estressores no ambiente de trabalho (FONSECA, MELLO, 2016).

O levantamento bibliográfico de estudos relacionados à Síndrome de Burnout mostra-se relevante para a comunidade científica, trabalhadores e para sociedade visto que possibilita a investigação da prevalência da Síndrome nos profissionais bem como aos aspectos de riscos que contribuem para o seu surgimento.

Nesse intuito, o presente estudo teve como objetivo revisar a produção científica nacional contextualizando a Síndrome de Burnout em enfermeiros de unidade de terapia intensiva.

\section{MÉTODO}

Trata-se de uma revisão integrativa de literatura, mediante pesquisa bibliográfica eletrônica, realizada no período entre maio e julho de 2017, na Biblioteca Virtual de Saúde (BVS) a partir de periódicos indexados nas bases de dados LILACS, IBECS, MEDLINE, BDENF e Index Psicologia.

Para elaboração desta revisão integrativa foram delimitadas as etapas a seguir: identificação do tema e seleção da hipótese ou questões de pesquisa para a elaboração da revisão integrativa; definição dos critérios para inclusão e exclusão dos artigos científicos e das informações a serem extraídas; 
categorização e avaliação dos estudos incluídos na revisão; interpretação dos resultados; e apresentação da revisão/síntese do conhecimento.

Considerando o objetivo proposto, a revisão do estado da arte foi norteada pelo seguinte questionamento: o que tem sido estudado no cenário nacional acerca da Síndrome de Burnout em enfermeiros na UTI?

Para localização dos artigos foram utilizados os descritores: enfermeiros, Burnout, unidade de terapia intensiva. Para seleção do material empírico foram adotados como critérios de inclusão artigos que abordassem a Síndrome de Burnout em Enfermeiros de unidade de terapia intensiva, disponíveis em língua portuguesa e inglesa; acessíveis na íntegra e publicada entre os anos de 2013 a 2017.

Assim, foram localizados 73 artigos relacionados à temática, dos quais, 5 preencheram aos critérios de inclusão estabelecidos, conforme detalhado na Figura 1.

Figura 1 - Fluxograma do percurso metodológico utilizado na revisão integrativa

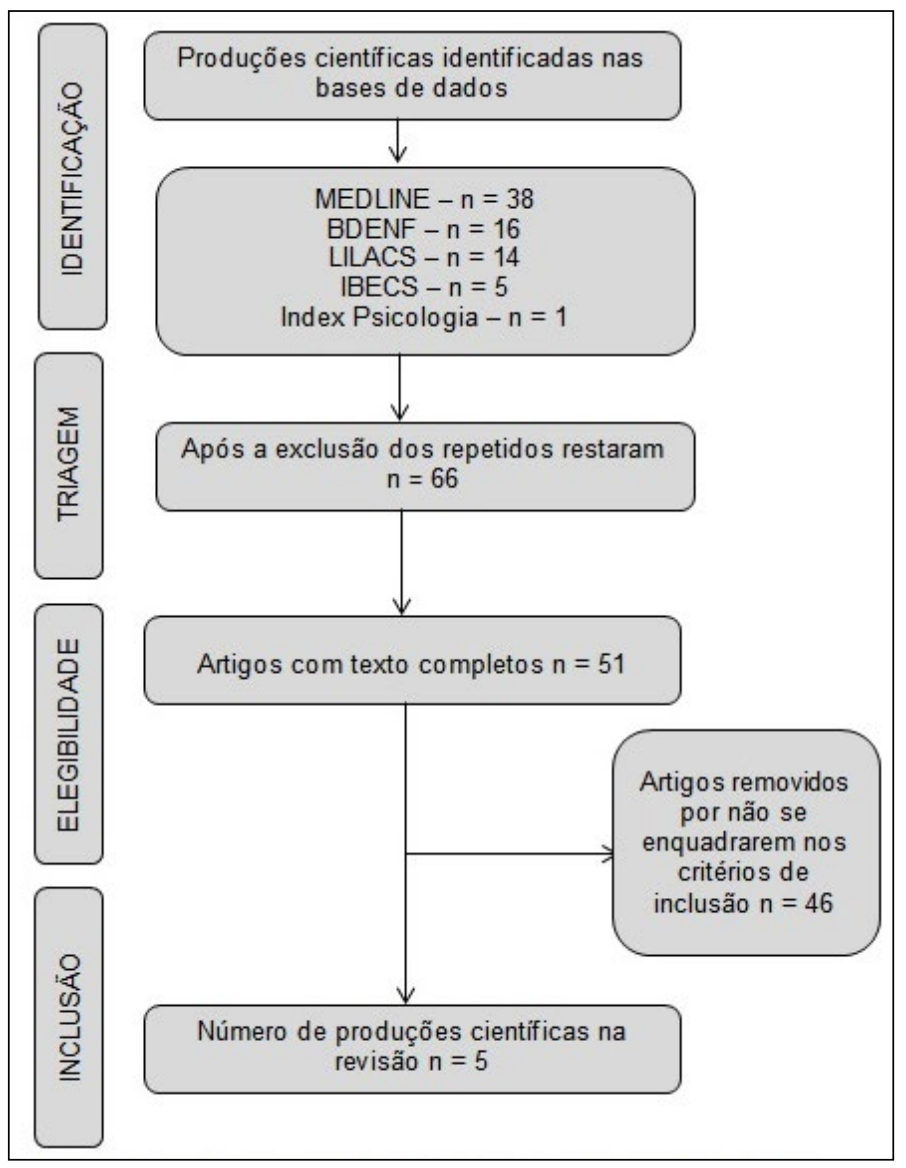

Fonte: Dados da pesquisa, 2017. 


\section{RESULTADOS E DISCUSSÃO}

A literatura traz que UTI, embora seja o local onde atende pacientes graves agudos e recuperáveis, é um dos ambientes mais agressivos, tensos e traumatizantes do hospital. Esses fatores agressivos atingem também toda a equipe multiprofissional, principalmente a Enfermagem, sendo grande a probabilidade de que esses profissionais estejam expostos aos mais variados fatores associados ao estresse. Dentre os fatores que corroboram a decorrência de tais complexidades estão a estrutura física, conviver em um local fechado com membros da equipe, o barulho constante, os equipamentos de alta tecnologia, a movimentação intensa de pessoas, e sofrimento dos pacientes (DECEZARO et al., 2014).

A Síndrome de Burnout apresenta-se como um acometimento multidimensional e acarreta prejuízos na atividade laboral de profissionais. A tabela 1 apresenta uma síntese dos estudos que integraram esta revisão, segundo autores, ano, tipo e objetivos do estudo.

Tabela 1. Síntese dos estudos sobre Síndrome de Burnout em enfermeiros de unidades de terapia intensiva. $(n=5)$

\begin{tabular}{|c|c|c|c|}
\hline $\begin{array}{c}\text { Autores } \\
\text { Ano }\end{array}$ & Título & Tipo do estudo & Objetivo do estudo \\
\hline $\begin{array}{l}\text { Martins, C. C. F., } \\
\text { Dantas, M. S. P., } \\
\text { Marinho, F. P., } \\
\text { Almeida, L. A., } \\
\text { Santos, V. E. P. } \\
\text { (2014). }\end{array}$ & $\begin{array}{l}\text { Síndrome de } \\
\text { Burnout em } \\
\text { enfermeiros } \\
\text { atuantes em } \\
\text { unidade de terapia } \\
\text { intensiva. }\end{array}$ & $\begin{array}{l}\text { Estudo descritivo- } \\
\text { exploratório } \\
\text { com abordagem } \\
\text { quantitativa do tipo } \\
\text { corte transversal. }\end{array}$ & $\begin{array}{l}\text { Verificar a ocorrência da } \\
\text { Síndrome de Burnout em } \\
\text { enfermeiros que prestam } \\
\text { assistência a pacientes } \\
\text { internados nas unidades de } \\
\text { terapias intensivas (UTI) dos } \\
\text { hospitais de ensino da cidade } \\
\text { de Teresina, Piaui. }\end{array}$ \\
\hline $\begin{array}{l}\text { Fonseca, T. C. P., } \\
\text { Mello, R. (2016). }\end{array}$ & $\begin{array}{l}\text { Síndrome de } \\
\text { Burnout entre } \\
\text { profissionais } \\
\text { de enfermagem } \\
\text { de unidades } \\
\text { intensivas em um } \\
\text { hospital público. } \\
\end{array}$ & $\begin{array}{l}\text { Pesquisa de } \\
\text { campo, com } \\
\text { caráter descritivo } \\
\text { e exploratório } \\
\text { e abordagem } \\
\text { quantitativa. }\end{array}$ & $\begin{array}{l}\text { Determinar a prevalência da } \\
\text { Síndrome de Burnout entre } \\
\text { profissionais de enfermagem } \\
\text { das unidades intensivas de um } \\
\text { hospital público. } \\
\text { Identificar fatores estressores } \\
\text { no ambiente de trabalho. }\end{array}$ \\
\hline $\begin{array}{l}\text { Decezaro, A., } \\
\text { Frizon, G., Silva, } \\
\text { O. M., Tiniollo, C. } \\
\text { L., Busnello, G. } \\
\text { F., Ascari, R. A. } \\
\text { (2014). }\end{array}$ & $\begin{array}{l}\text { O estresse dos } \\
\text { enfermeiros } \\
\text { que atuam na } \\
\text { unidade de terapia } \\
\text { intensiva: } \\
\text { uma revisão de } \\
\text { literatura. }\end{array}$ & $\begin{array}{l}\text { Este estudo } \\
\text { constitui-se em } \\
\text { uma revisão de } \\
\text { literatura. } \\
\\
\end{array}$ & $\begin{array}{l}\text { Identificar iniciativas para } \\
\text { enfrentar o estresse laboral } \\
\text { que acomete a enfermagem da } \\
\text { unidade de terapia intensiva. }\end{array}$ \\
\hline
\end{tabular}




\begin{tabular}{|c|c|c|c|}
\hline $\begin{array}{c}\text { Autores } \\
\text { Ano }\end{array}$ & Título & Tipo do estudo & Objetivo do estudo \\
\hline $\begin{array}{l}\text { Campos, J. F., } \\
\text { David, H. M. S. L, } \\
\text { Souza, N. V. D. O. } \\
(2014) .\end{array}$ & $\begin{array}{l}\text { Prazer e } \\
\text { sofrimento: } \\
\text { avaliação de } \\
\text { enfermeiros } \\
\text { intensivistas à luz } \\
\text { da psicodinâmica } \\
\text { do trabalho. }\end{array}$ & $\begin{array}{l}\text { Estudo exploratório } \\
\text { de abordagem } \\
\text { quantitava e } \\
\text { desenho transversal, } \\
\text { descritivo e } \\
\text { inferencial }\end{array}$ & $\begin{array}{l}\text { Analisar, mensurar e avaliar } \\
\text { os riscos de adoecimento } \\
\text { relacionados ao trabalho do } \\
\text { enfermeiro } \\
\text { de UTI. }\end{array}$ \\
\hline $\begin{array}{l}\text { Melo, E. M. V. B., } \\
\text { Santos, R. L. N., } \\
\text { Cavalcante, C. A. } \\
\text { T., Siqueira, D. } \\
\text { G., Medeiros, L. } \\
\text { P., Sousa, D. A. } \\
\text { (2014). }\end{array}$ & $\begin{array}{l}\text { Prevalência da } \\
\text { Síndrome de } \\
\text { Burnout nas UTI's } \\
\text { em Enfermeiros } \\
\text { de um Hospital } \\
\text { Escola do Recife. }\end{array}$ & $\begin{array}{l}\text { Estudo descritivo } \\
\text { do tipo corte } \\
\text { transversal } \\
\text { prospectivo, de } \\
\text { abordagem } \\
\text { quantitativa. }\end{array}$ & $\begin{array}{l}\text { Caracterizar a amostra e } \\
\text { determinar a prevalência } \\
\text { da Síndrome de Burnout em } \\
\text { enfermeiros que trabalham em } \\
\text { UTI' de um hospital escola do } \\
\text { Recife. }\end{array}$ \\
\hline
\end{tabular}

Silva, Avelino, Gouveia et al. (2014) em seu estudo aplicou instrumentos com 33 sujeitos, para avaliação das três dimensões sintomatológicas da identificação da Síndrome de Burnout em enfermeiros da UTI. Constatou-se que a prevalência dos sujeitos participantes é do sexo feminino ( $94 \%)$, com idades entre 22 a 40 anos (64\%) e 41 a 62 anos (36\%). Em relação ao estado civil predominou a porcentagem de casados $(52 \%)$, seguidos pelos solteiros $(36 \%)$, além disso, $70 \%$ da amostra relataram ter filhos.

A prevalência de mulheres no levantamento corrobora com o estudo de Fonseca e Mello (2016), no qual, dos 22 sujeitos participantes, 22,7\% eram do sexo masculino e $77,2 \%$ do sexo feminino, com uma média de idade de 45,2 anos, principalmente solteiros, e 59\% dos profissionais possuem filhos.

Decezaro et al. (2014), relata que o risco para adquirir a síndrome de Burnout diminui a medida que o longo período de trabalho se aproxima, estes apresentaram um baixo nível de estresse. Já no estudo de Melo et al. (2014), o alto nível de exaustão emocional, é possível perceber que a categoria mais afetada é a de enfermeiros que trabalham há mais de 21 anos, possuem carga horária maior que 60 horas semanais.

Com relação às características dos sintomas somáticos decorrentes do trabalho, detectou-se que os mais frequentes foram: dores nos ombros ou nuca $(86 \%)$, cefaleia $(71 \%)$, pouco tempo para si mesmo $(67 \%)$, dificuldade de memória e concentração (57\%), cansaço mental $(57 \%)$, irritabilidade fácil, fadiga generalizada e perda de senso de humor (52\%) e das dimensões sintomatológicas a despersonalização, que é aquele que demonstra que o profissional apresenta-se insensível diante dos acontecimentos no serviço, foi o fator que mais prevaleceu. Nesse levantamento, foi possível detectar a ocorrência da síndrome de Burnout em 2 enfermeiros, correspondendo a um percentual de $6 \%$, ambas eram do sexo feminino, com faixa etária entre 22 a 40 
anos, sem filhos, sendo uma casada e a outra solteira, quanto a atividade física ambas praticavam exercícios (SILVA et al., 2014).

A exaustiva carga horária de trabalho semanal contribui para o surgimento de sintomatologia estressante. No estudo de Melo et al. (2014), os profissionais possuem uma carga horária semanal de trabalho superior a 60 horas $(54,5 \%)$ somando-se todas as suas atividades laborais.

Segundo Melo et al. (2014), foi identificado escore positivo para Síndrome de Burnout em 6 enfermeiros representando 15,4\% dos pesquisados, esse valor é considerado baixo em se tratando de profissionais que lidam com pessoas e pacientes com eminente risco de morte requerendo uma atenção especial. Com risco de desenvolver a síndrome de Burnout, 29 (74,3\%) dos enfermeiros estão dentro desse limite, e 4 (10,3\%) enfermeiros estão fora desse risco. Profissionais que não recebeu diagnóstico ou característica especifica para Síndrome de Burnout, apresentaram risco para desenvolver a síndrome devida às condições estressoras na qual estão impostas (DECEZARO et al., 2014).

Reforça a necessidade de realização de estratégias para à valorização dos trabalhadores e o investimento na educação permanente em saúde, as quais podem ter papel protetor para a saúde do trabalhador contra estressores no cotidiano de trabalho, à medida que proporcionam autonomia aos trabalhadores de enfermagem.

\section{CONCLUSÃO}

Este estudo contribui para uma melhor compreensão da subjetividade sobre a Síndrome de Burnout no trabalho de enfermagem. Bem como, denotam os fatores que influenciam o profissional no ambiente em que está inserido, alterando seu processo de trabalho e sua saúde física e mental.

Torna-se importante o uso de estratégias de redução do estresse físico, psíquico e emocional que ameaçam a adaptação do profissional de enfermagem na UTI, para que assim desenvolvam mecanismos de enfrentamento para lidar efetivamente com os fatores estressores que predispõem ao desenvolvimento da Síndrome de Burnout.

Diante disso, percebe-se a necessidade de reestruturações organizacionais e mudança nas condições de trabalho, abrindo espaços institucionais para discussões dos fatores que predispõe o desenvolvimento da síndrome de Burnout.

O profissional de enfermagem necessita iniciar a mudança, tornandose mais saudáveis e capazes de compreender seus limites de trabalho na UTI. Isso pode ser justificado devido a grande demanda de atribuições inerentes ao Enfermeiro, a carga horária exaustiva, a quantidade de vínculos empregatícios, o emocional envolvido no cuidado direto ao paciente, dentre outros fatores relacionados à prática profissional. 
Com isso, nota-se a importância em compreender e modificar as condutas voltadas a saúde do trabalhador, enfatizando na prevenção de doenças, principalmente no que se refere às ações que podem contribuir para minimizar fatores estressores que podem desencadear a Síndrome de Burnout.

A qualidade de vida e a realização profissional devem compor um ciclo vital como fator positivo no processo de trabalho da equipe de enfermagem, contribuindo para uma melhor assistência oferecida seja no ambiente assistencial, como no gerencial.

\section{REFERÊNCIAS}

ALKIMIM, C. F. C.; Prado, B. M. P.; Carreiro, D. L. et al. Fatores associados à Síndrome de Burnout entre profissionais intensivistas de hospital universitário. Tempus, actas de saúde colet, v.8, n.4, p.157-176, 2014. Disponível em: http://dx.doi. org/10.18569/tempus.v8i4.1590

CAMPOS, J. F.; DAVID, H. M. S. L.; SOUZA, N. V. D. O. Prazer e sofrimento: avaliação de enfermeiros intensivistas à luz da psicodinâmica do trabalho. Escola Anna Nery Revista de Enfermagem. v.18, n.1, 2014. Disponível em: http:/ /dx.doi. org/10.5935/1414-8145.20140013

CARVALHAIS, F. R.; AGUILAR, A. M. M.; MENDONÇA, R. L.; et al. Frequência da síndrome de burnout em uma unidade de terapia intensiva: uma perspectiva multiprofissional. Rev. Pre. Infec e Saúde, v.1, n.4, p.1-10, 2015. Disponível em: http://www.ojs.ufpi.br/index.php/nupcis/article/view/4271/pdf

DECEZARO, A.; FRIZON, G.; SILVA, O. M.; et al. O Estresse dos Enfermeiros que atuam na Unidade de Terapia Intensiva: uma Revisão de Literatura. Revista UNINGÁ Review. v.19, n.2, p.29-32, 2014. Disponível em: https://www. mastereditora.com.br/periodico/20140801_093235.pdf

FERNANDES, L. S.; NITSHER, M. J. T.; GODOY, I. Fatores preditores da síndrome de burnout em enfermagem na Unidade de terapia intensiva. Rev enferm ufpe on line. Recife, v.9, n.5, p.8374-8, 2015. Disponível em: https://doi.org/10.5205/1981-8963v9i5a10602p8374-8378-2015

FERREIRA, G. B.; ARAGÃO, A. E. A.; OLIVEIRA, P. S. Síndrome de burnout na enfermagem Hospitalar/intensivista: o que dizem os Estudos? Sanare, Sobral. v.16, n.01, p.100-108, 2017. Disponível em: https://sanare.emnuvens.com.br/sanare/ article/view $/ 1100$

FONSECA, T. C. P.; MELLO, R. Síndrome de Burnout entre Profissionais de Enfermagem de Unidades Intensivas em um Hospital Público. Rev enferm UFPE online. v.10, n.1, p.296-303, 2016. Disponível em: https:/ /doi.org/10.5205/1981-8963v10i1a10953p296-303-2016 
MARTINS, C. C. F.; DANTAS, M. S. P.; MARINHO, F. P.; et al. Agentes estressores na terapia intensiva: visão dos profissionais de enfermagem. Rev enferm, UFPE on line. v.8, n.10, p.3386-91, 2014. Disponível em: https:/ / doi.org/10.5205/1981-8963v8i10a10070p3386-3392-2014

MELO, E. M. V. B., SANTOS, R. L. N., CAVALCANTE, C. A. T.; et al.. Prevalência da Síndrome de Burnout nas UTI's em Enfermeiros de um Hospital Escola do Recife. Revista de Psicologia. 8(24), 2014. Disponível em: doi: https:/ /doi.org/10.14295/ idonline.v8i24.306

MONTEIRO, J. K.; OLIVEIRA, A. L. L.; RIBEIRO, C. S.; et al. Adoecimento Psíquico de Trabalhadores de Unidades de Terapia Intensiva. Psicologia ciência e profissão. v.33, n.2, p.366-379, 2013. Disponível em: https://dialnet.unirioja.es/servlet/ articulo?codigo $=6123174$

PEREIRA, S. M., TEXEIRA, C. M., RIBEIRO, O.; et al. Burnout em médicos e enfermeiros: estudo quantitativo e multicêntrico em unidades de cuidados paliativos em Portugal. Revista de Enfermagem Referência. v.4, n.3, p.55-64, 2014. Disponível em: http:/ / dx.doi.org/10.12707/RIII13178

SCHMIDT, D. R. C.; PALADINI, M.; BIATO, C.; et al. Qualidade de vida no trabalho e burnout em trabalhadores de enfermagem de Unidade de Terapia Intensiva. Rev Bras Enferm. v.66, n.1, p.13-7, 2016. Disponível em: http:/ /dx.doi.org/10.1590/S003471672013000100002

SILVA, G. K. C.; AVELINO, F. V. S. D.; GOUVEIA, M. T. O.; et al. Síndrome de Burnout em enfermeiros atuantes em unidade de terapia intensiva. Enferm. Foco, v.5, n.3/4, p.75-78, 2014. Disponível em: http:/ / revista.cofen.gov.br/index.php/ enfermagem/article/view/563/245

SILVA, J. L. L., SOARES, R. S., COSTA, F. S., et al. (. Psychosocial factors and prevalence of burnout syndrome among nursing workers in intensive care units. Rev Bras Ter Intensiva, v.27, n.2, 2015. Disponível em: http:/ / dx.doi.org/10.5935/0103507X.20150023 